\title{
Study on the effect of intestinal immunity that the neonatal piglet co-infection with porcine deltacoronavirus and porcine epidemic diarrhoea virus
}

\section{Jixiang Liang ( $\nabla 877581522 @ q q . c o m$ )}

Huazhong Agriculture University: Huazhong Agricultural University https://orcid.org/0000-0002-15318332

\section{Yang Li}

Huazhong Agriculture University

\section{Zhishan Yan}

Huazhong Agricultural University College of Plant Science and Technology

\section{Zhe Jiao}

Huazhong Agriculture University

\section{Dapeng Peng}

Huazhong Agriculture University

\section{Wanpo Zhang}

Huazhong Agriculture University

\section{Research Article}

Keywords:

Posted Date: January 20th, 2022

DOI: https://doi.org/10.21203/rs.3.rs-1152562/v1

License: (c) (i) This work is licensed under a Creative Commons Attribution 4.0 International License. Read Full License 


\section{Abstract}

Porcine deltacoronavirus (PDCoV) and porcine epidemic diarrhoea virus (PEDV) have been often simultaneously detected in coronavirus diarrhea piglets. But the intestinal immune of the interaction between the co-circulating PDCoV and PEDV are unknown. Therefore, the study was conducted to investigate intestinal immunity of neonatal piglets that were exposed with PDCoV first and then PEDV subsequently. The amount and distribution of $\mathrm{CD}^{+} \mathrm{T}$ lymphocytes, $\mathrm{B}$ lymphocytes and goblet cells (GCs) in small intestine were analyzed by immunohistochemistry and periodic acid-schiff respectively. The expression of pattern recognition receptors and downstream mediator genes were analyzed by qPCR. The results showed that the number of GCs, $\mathrm{CD}^{+} \mathrm{T}$ lymphocytes and $\mathrm{B}$ lymphocytes in the small intestine of the PDCoV + PEDV co-inoculated piglets were increased compared with PEDV single-inoculated piglets. The piglets in the group of PDCoV + PEDV were significantly up-regulated IFN- $a$ and IFN- $\lambda_{1}$ when compared with the PEDV single-inoculated piglets. These results suggest that the PDCoV + PEDV coinfected piglets can superiorly activate intestinal antiviral immunity compared to PEDV single-infected piglets, which provide a new insight into the pathogenesis mechanism of swine enteric coronavirus coinfection that may be used for vaccination in the future.

\section{Introduction}

Currently, the viral porcine diarrhea, mainly caused by coronavirus such as porcine epidemic diarrhea virus (PEDV) and porcine deltacoronavirus (PDCoV), have been continued to spread and caused serious economic losses in pig farms [1]. PEDV and PDCoV were first reported in the United Kingdom [2] and in Hong Kong [3] respectively, and subsequently detected from pigs in other countries. PDCoV or PEDV is enveloped, single-stranded, positive-sense RNA viruses that belong to the family Coronaviridae [4]. These two viruses can be transmitted through the fecal oral route and primarily infect small intestinal epithelial cells [4]. These leads to indistinguishable clinical signs characterized by severe diarrhoea, anorexia, dehydration, and weight loss [5]. But the mortality and the intestinal lesion caused by the PDCoV infected piglets were relatively milder than the PEDV infected piglets [6, 7].

Previous studies reported that coinfection with PEDV up to 50\% in PDCoV infected diarrhoeal pigs [7, 8]. Some studies have shown that simultaneously infected of PDCoV and PEDV enhances the disease severity in piglets $[4,5]$. While, one study has shown that no increased severity of PEDV infectivity by PDCoV co-infection, when first inoculated with virulent PEDV PC21A followed in $16 \mathrm{~h}$ by PDCoV OH-FD22, or they are simultaneously inoculated with the PEDV and PDCoV strains [9]. Meanwhile, a similar result also has been reported in our previous study, coinfection with PDCoV first and then PEDV has no exacerbate the disease severity compared to a single infection with either virus [10]. Thus, it is controversial the synergistic pathogenic mechanisms of PDCoV and PEDV, and the detailed mechanism by which that co-infection impact on the intestinal immunity remains unclear.

It is widely known that the intestinal mucosa often plays as the first line of defense against pathogenic microorganisms [11]. Intraepithelial lymphocytes and lamina propria lymphocytes play a key role in either 
maintaining or restoring intestinal immune homeostasis [12]. The intestinal mucosal surface is covered mucus layer, which is mainly consist of mucins secreted by goblet cells (GCs), and for both lubrication and barrier function of the intestinal epithelium against enteric pathogens $[13,14]$. As pattern recognition receptors $\triangle P R R s \rrbracket$, toll like receptors (TLRs) can recognize various pathogens and mediate the innate immune response of host defense [15]. Upon virus infects the host, the PRRs to recognize thoses viruses to the production of interferons (IFNs), which are the main antiviral cytokine involved in innate immunity [16]. IFNs are divided into three types (types I, II, and III) based on their different receptors, such as IFN-a and IFN- $\lambda_{1}$ are play an important role in antiviral immunity [17]. Notably, one study showed that PDCoV infected piglets significantly induced the gene expression of IFN- $a$ in the small intestine, while another study showed that PEDV infected piglets suppressed of IFN-a in the small intestine [18, 19]. In addition, studies reported that IFN- $\lambda_{1}$ preferably inhibits PEDV infection of porcine intestinal epithelial cells compared with IFN-a [20].

In order to further study the effect of intestinal immunity that the PDCoV + PEDV coinfected piglets. We investigated the number and distribution of immune cells by immunohistochemical (IHC) and periodic acid-schiff (PAS) in the small intestine. Also observed the genes expression profile of pattern recognition

receptors and downstream mediator cytokines were analyzed by RT-qPCR. Our results assessed the effect on the intestinal immune of PDCoV + PEDV coinfected piglets.

\section{Materials And Methods}

\section{Virus strains and piglets}

PDCoV-CHN-HG-2017 strain (accession number MF095123) and PEDV-JS-A strain (accession number MH748550) were used in the study. These two viruses were from Dr. Qigai He and Dr. Zili Li of the State Key Laboratory of agricultural microbiology, Huazhong Agricultural University, respectively. Twelve neonatal non-colostrum-fed piglets were selected from a PEDV, TGEV, PRCV and PDCoV negative herd in Wuhan.

\section{Experimental design}

Twelve piglets were divided randomly into 4 groups (3/group): group of PEDV, group of PDCoV, group of PDCoV + PEDV (co-infection), and group of uninfected control (mock). Piglets were housed in separated rooms by treatment with strict biosecurity and biocontainment. The piglets were fed milk substitute (Anyou, China) every $4 \mathrm{~h}$. Piglets were acclimated for $48 \mathrm{~h}$ before inoculation. On hour 0 post-inoculation $(\mathrm{HPI})$, the piglets in the groups of PDCoV and PDCoV + PEDV were exposed orally with $5 \mathrm{ml}$ of $1 \mathrm{x}$ $10^{5} \mathrm{TCID}_{50} / \mathrm{mL}$ PDCoV-CHN-HG-2017/piglet, while the piglets in the groups of PEDV and uninfected control were exposed orally with $5 \mathrm{~mL}$ maintenance medium. At $48 \mathrm{HPI}$, the piglets in the groups of PEDV and PDCoV + PEDV were inoculated orally with $4 \mathrm{ml}$ of $1 \times 10^{5} \mathrm{TCID}_{50} / \mathrm{mL}$ PEDV-JS-A/piglet, while the piglets in the groups of PDCoV and uninfected control were inoculated orally with an equal volume of maintenance medium. Piglets were euthanized for pathologic examination at the end study of $72 \mathrm{~h}$ post 
of 2 nd exposure, as described previously([10]). One part of tissue samples of proximal duodenum, middle jejunum and distal ileum were fixed with $10 \%$ formalin for $48 \mathrm{~h}$, and then dehydrated, paraffin-embedded, made into $4 \mu \mathrm{m}$ serial slices. The other part of middle jejunum tissue samples was stored at $-80^{\circ} \mathrm{C}$ for molecular biological analysis.

\section{Periodic Acid Schiff (PAS) stain}

To evaluate and quantify goblet cells, sections were stained strictly according to the instructions of Periodic Acid-Schiff kit (L/N. G1008-100ML, Servicebio). Six fields were randomly selected from each tissue section for microscopic observation, and the number of GC in villous epithelium was counted by Image-Proplus.

\section{Immunohistochemistry (IHC)}

For immunohistochemistry, the slides were deparaffinized, rehydrated, and treated with $3 \% \mathrm{H}_{2} \mathrm{O}_{2}$. Tissue slides were immersed in antigen repair buffer for thermal repair, and $5 \%$ goat serum was sealed at room temperature. Appropriate volume of primary antibody solution was used to cover the tissue at $4{ }^{\circ} \mathrm{C}$ overnight, antigen repair buffer and primary antibody solution in Table 1. After rinsing with PBS, the slides were incubated with HRP-conjugated goat anti-rat/rabbit IgG (GK600711A, Gene tech, China) at room temperature for 30 min and then were visualized with DAB Detection Kit (GK500710, Gene Tech, China). The slides were observed under a microscope to detect positive signal particles, showing brown precipitation in the cells. The sections were stained with hematoxylin. Observed and collected images under optical microscope.

\section{Real-time Fluorescent quantitative PCR}

Total RNA was extracted from the jejunum tissues (every piglet) with RNA-easy ${ }^{\mathrm{TM}}$ Isolation Reagent (L/N.7E371G9, Vazyme, China), cDNA was synthesized from the extracted RNA by reverse transcription using an HiScript § II Q RT Super Mix kit (L/N.7E421E0, Vazyme, China), and real-time RT-qPCR was performed using the Chamq Universal SYBR Master Mix (L/N.7E472E0, Vazyme, China). The levels of TLR2, TLR3, MAVS, IRF3, MDA5, IFN-a, IFN- $\lambda_{1}$ and NF-kb were detected by Real-Time PCR with the primers listed in Table 2. The cycling parameters were $95^{\circ} \mathrm{C}$ for $10 \mathrm{~min} ; 40$ cycles at $94^{\circ} \mathrm{C}$ for $30 \mathrm{sec} ; 60^{\circ} \mathrm{C}$ for 1 min. The specific pig gene primers were designed on NCBI and synthesized by Sangon Biotech Corporation (Wuhan, China).

\section{Statistical Analysis}

All Small intestine tissues PAS and IHC sections were examined with the Nikon 80i microscope (Nikon, Japan) equipped with the Nikon 80i camera and Image Scope x64 software (Leica, Germany). Data presented as Mean \pm SEM. All data were analyzed with Prism 8.0 (GraphPad Software Inc). Two-way ANOVA were used to determine significant differences among different groups. $\mathrm{P}<0.05$ was considered statistically significant. 


\section{Results}

\section{Analysis of $\mathrm{CD3}^{+} \mathrm{T}$ lymphocytes in small intestine of piglets}

Immunohistochemistry demonstrated that CD3 antigen positive expressed in villi lamina propria, crypts and peyer's patches in the small intestine (Fig. 1a to I). Image analysis demonstrated that CD3 antigen in small intestine of all viral infected piglet groups were lower than the piglets in the group of uninfected control, with the difference was significant in duodenum $(P<0.001$, Fig. $1 \mathrm{~m})$ and jejunum $(P<0.001$, Fig. 1n), but the difference was no significant in ileum ( $P>0.05$, Fig. 10). Image analysis demonstrated that CD3 antigen in duodenum $(P<0.05$, Fig. $1 \mathrm{~m})$ of the piglets in the group of PEDV was significantly lower than the piglets in the groups of PDCoV and PDCoV + PEDV. On the contrary, the piglets in the groups of PDCoV and PDCoV + PEDV presented with a lower CD3 antigen positivity in the ileum $(P>0.05$, Fig. 10) compared with the piglets in the group of PEDV. The piglets in the group of PEDV presented with a significant lower CD3 antigen positivity in the jejunum compared with the piglets in the group of PDCoV + PEDV $(P<0.01$, Fig. 1n), and not presented with a significant lower CD3 antigen positivity in the jejunum compared with the piglets in the group of PDCoV ( $>>0.05$, Fig. 1n). Image analysis demonstrated that CD3 antigen of the piglets in the group of PDCoV was lower compared PDCoV + PEDV coinfected piglets in jejunum $(P<0.05$, Fig. $1 \mathrm{n})$ and ileum $(P>0.05$, Fig. 10), but was minor higher in duodenum $(P>0.05$, Fig. $1 \mathrm{~m})$.

\section{Analysis of B lymphocytes in small intestine of piglets}

CD20 is a specific membrane antigen of B lymphocytes [21]. Immunohistochemistry showed that positive CD20 antigen positive mainly expressed in intestinal villus lamina propria, submucosa and peyer's patches of small intestine (Fig.2 a to I). Image analysis demonstrated that CD20 antigen in ileum of all viral infected piglet groups were lower than the piglets in the group of uninfected control $(P<0.001$, Fig. 2 o), but were minor higher in jejunum ( $P>0.05$, Fig. $2 \mathrm{n}$ ). Image analysis demonstrated that CD20 antigen in duodenum of all uninfected control piglets were lower than the piglets in the groups of PEDV $(P>0.05$, Fig. $2 \mathrm{~m}$ ) and PDCoV + PEDV $(P<0.01$, Fig. $2 \mathrm{~m})$, but were minor higher the piglets in the groups of PDCoV $(P>0.05$, Fig. $2 \mathrm{~m})$. Image analysis demonstrated that $C D 20$ antigen positivity in duodenum $(P<0.05$ or $P$ $<0.001$, Fig. $2 \mathrm{~m}$ ) and jejunum ( $\mathrm{P}>0.05$, Fig. $2 \mathrm{~m}$ ) of the piglets in the group of PDCoV + PEDV was higher than the piglets in the groups of PDCoV and PEDV. On the contrary, the piglets in the group of PDCoV + PEDV presented with a lower CD20 antigen positivity in the ileum compared with the piglets in the groups of PEDV and PDCoV ( $P>0.05$ or $P<0.01$, Fig. $2 \mathrm{~m}$ ). Image analysis demonstrated that CD20 antigen positivity of the piglets in the group of PDCoV was minor lower compared PEDV single infected piglets in duodenum ( $P>0.05$, Fig. $2 \mathrm{~m}$ ) and jejunum $(P>0.05$, Fig. $2 \mathrm{n})$, but was significantly higher in duodenum $(P<0.05$, Fig.2 o).

\section{Analysis of Goblet cells in small intestine of piglets}


Goblet cells were purplish red stained by PAS stain, and mainly distributed in villi and crypt of small intestine (Fig.3 a to I). Image analysis demonstrated that the number of GCs in small intestine of all viral infected piglet groups were lower than the piglets in the group of uninfected control $(P>0.05, P<0.01$ or $P<0.001$, Fig. $3 \mathrm{~m}$ to $\mathrm{o}$ ). Image analysis demonstrated that the number of GCs of the piglets in the groups of PDCoV and PDCoV + PEDV were higher compared PEDV single infected piglets in duodenum $(\mathrm{P}<$ 0.001 , Fig. $3 \mathrm{~m})$ and jejunum $(P<0.001$ or $P>0.05$, Fig. $3 \mathrm{n})$, but were lower in ileum $(P<0.001$ or $P>0.05$, Fig.3 o). Image analysis demonstrated that the number of GCs of the piglets in the group of PDCoV was higher compared PDCoV + PEDV co-infected piglets in duodenum $(P>0.05$, Fig. $3 \mathrm{~m})$ and jejunum $(P<$ 0.01 , Fig. $3 \mathrm{n})$, but was significantly lower in ileum $(P<0.001$, Fig. 3 o).

\section{Expression of cytokines related to intestinal pattern recognition receptors}

PDCoV + PEDV infected piglets revealed significant higher TLR2, TLR3, MAVS and IFN-a mRNA than the piglets in the groups of PEDV, PDCoV and uninfected control $(P<0.01$ or $P<0.001$, Fig.4). PDCoV infected piglets showed significant higher TLR3, IRF3 and MAVS mRNA than the piglets in the groups of PEDV and uninfected control $(P<0.001$, Fig.4). PDCoV + PEDV infected piglets revealed significant higher IRF3 mRNA than the piglets in the groups of PEDV and uninfected control $(P<0.001$, Fig.4). PDCoV infected piglets revealed significant lower MDA-5, IFN- $\lambda 1$ and IFN- $a$ mRNA than the piglets in the group of uninfected control $(P<0.01$ or $P<0.001$, Fig.4). PDCoV infected piglets showed significant lower IFN- $\lambda 1$ mRNA than the piglets in the group of PDCoV + PEDV ( $<<0.05$, Fig.4). PEDV infected piglets showed a significant lower NF-kb mRNA than the piglets in the groups of uninfected control and PDCoV + PEDV (P $<0.001$, Fig.4). Piglets in the group of uninfected control were significantly higher IFN- $\lambda 1 \mathrm{mRNA}$ than the piglets in the groups of PEDV and PDCoV $(P<0.001$, Fig.4).

\section{Discussion}

In previous study, the pathogenicity of two virus have been analyzed, 5-day-old piglets were orally inoculated with $10 \mathrm{~mL}$ of $1 \times 10^{6} \mathrm{TCID}_{50} / \mathrm{mL}$ PDCoV-CHN-HG-2017 or $3 \mathrm{~mL}$ of $1 \times 10^{5} \mathrm{TCID}_{50} / \mathrm{mL}$ PEDV-JS$A$, which were consistent with the results of this study $[22,23]$. Meanwhile, our previous reports showed that an animal model of PDCoV + PEDV coinfection was successfully constructed in neonatal noncolostrum-fed piglets, and demonstrated that coinfection of PDCOV and PEDV can alter PEDV tropism changes from epithelial cells of small intestine to gastric epithelial cells and macrophages in Peyer's patches in the ileum, and coinfection with PDCoV and PEDV are showed significant lower viral loads in the small intestine compared to a single infection with either virus [10]. However, these experimental results need to be verified whether they are related to immune cells in the intestine.

The intestinal mucosal immune system plays as the first line of defense against pathogenic microorganisms [11]. Most intestinal intraepithelial lymphocytes are $C D 3^{+} T$ cells, and distribute in the intestinal epithelium and subjacent superficial lamina propria, and thus are positioned at the border of the microbial and dietary environment [24]. B lymphocytes cells also are major immune cells found in the intestinal mucosa, and mainly produce IgA antibodies [25]. And intestinal immune cells or secreting 
cytokines are involved in either maintaining or regulating the immune homeostasis [11]. A study has showed that the number of IgA positive cells, $\mathrm{CD}^{+} \mathrm{T}$ cells decreased in the small intestine of TGEV infected piglets [24]. In this study, all infected piglets were decreased the number of $\mathrm{CD}^{+} \mathrm{T}$ lymphocytes in small intestine, when compared with all uninfected control piglets. At the same time, we notice that the $\mathrm{CD}^{+} \mathrm{T}$ lymphocytes in the PDCoV + PEDV coinfected piglets were higher than that in the piglets from the group of PEDV, especially in the duodenum and jejunum. However, all infected piglets increased the numbers of B lymphocytes except in the ileum, when compared with uninfected control piglets. A certain extent, these results showed that viruses may activate the antiviral immunity in the small intestine of all infected piglets, and B lymphocytes maybe migrate from ileal mucosa to duodenal mucosa and jejunal mucosa to secrete antibodies, especially in the PDCoV + PEDV co-infected piglets.

The mucus layer in the small intestine is mainly composed of mucin produced and secreted by the GCs, which is an important part of the intestinal mucosal immune system [13]. Previous research showed that during the early stages of PEDV infection of piglets, the numbers of GCs in the small intestine often significantly reduce [14]. As a result, the intestinal mucosal immune barrier of piglets was destroyed and secondary infections with other enteric pathogens may lead to increased mortality $[14,26]$. In this study, all infected piglets were decreased the numbers of GCs in the small intestine, and significantly decreased in the duodenum and jejunum, when compared with uninfected control piglets. This result showed that viral infection may decreased mucins that GCs secrete in the small intestine, and then possibly leading to an impaired mucus layer. At the same time, we notice that damaged of mucus layer the piglets in the group of PDCoV + PEDV co-infection was less than the piglets in the group of PEDV, especially in the duodenum.

Interferons could induce the expression of extensive range of antiviral effectors that help host to antagonize viral infections [27]. In this study, piglets in the groups of PDCoV or PEDV was suppressed the innate immunity of intestine, that is consistent with the previous reports $[4,18,28]$. Meanwhile, we found out the expression of IFN- $a$ and IFN- $\lambda_{1}$ in the PDCoV + PEDV infected piglets were significantly higher than that in PDCoV or PEDV single-inoculated piglets, and by analysis of TLRs signaling pathway in the small intestine. These results demonstrated that piglets infected with PDCoV first and then PEDV subsequently could activate intestinal antiviral immunity than that PEDV single-inoculated piglets. This may partly explain that our previous result PDCoV + PEDV co-infected piglets were showed significant lower PDCoV viral load or PEDV viral load in the small intestine compared a single infection of PDCoV or PEDV [10]. A similar study has reported that a possible interference or inhibition of PDCoV replication in the gastrointestinal tract coinfected with virulent PEDV strains, when first inoculated with virulent PEDV PC21A followed by PDCoV OH-FD22 [9]. In addition, another study also reported a similar result, IFN-a was significantly up-regulated in conventional 4-day-old piglets co-inoculated simultaneously with PEDV and PDCoV compared with PEDV or PDCoV singly inoculated piglets [4].

Taken together, those results showed that may activate the antiviral response of similar immune cells that in the small intestine after PDCoV infected piglets, which difficult for PEDV re-infected piglets. This 
may indicate that piglets are inoculated with inactivated PDCoV vaccine will also interfere with PEDV infection. However, this hypothesis needs to be further evaluated.

In conclusion, this study is the first report of infection with PDCoV first and then PEDV subsequently on mucosal immunity in neonatal piglets. Our results suggest that PDCoV + PEDV co-inoculated piglets were increased $B$ lymphocytes, $C D 3^{+} T$ lymphocytes and goblet cells compared with the PEDV single-inoculated piglets. In addition, IFN- $\alpha$ and IFN- $\lambda_{1}$ were significantly up-regulated in the PDCoV + PEDV co-inoculated piglets compared with PEDV single-inoculated piglets. These results suggest that the PDCoV + PEDV coinfected piglets can superiorly activate intestinal antiviral immunity compared to PEDV single infected piglets.

\section{Declarations}

Funding This work is supported by Da Bei Nong Group Promoted Project for Young Scholar of HZAU (Grant No. 2017DBN004).

Author contributions All authors contributed to the study conception and design. Material preparation, data collection and analysis were performed by Jixiang Liang, Yang Li, Zhe Jiao and Zhishan Yan. The first draft of the manuscript was written by Jixiang Liang and all authors commented on previous versions of the manuscript. All authors read and approved the final manuscript.

\section{Compliance with ethical standards}

Conflict of interest The authors declare that they have no competing interests.

Ethics approval All piglets used in the present study were humanely bred during the experiment and euthanized at the end of the experiment. Animal care and use protocols were reviewed and approved by the experimental animal monitoring committee of Huazhong Agricultural University.

\section{References}

1. Jia, S., Feng, B., Wang, Z., Ma, Y., Gao, X., Jiang, Y., Cui, W., Qiao, X., Tang, L., Li, Y., Wang, L., \& Xu, Y. (2019). Dual priming oligonucleotide (DPO)-based real-time RT-PCR assay for accurate differentiation of four major viruses causing porcine viral diarrhea. Molecular and cellular probes, 47, 101435. https://doi.org/10.1016/j.mcp.2019.101435

2. Wood E. N. (1977). An apparently new syndrome of porcine epidemic diarrhoea. The Veterinary record, 100(12), 243-244. https://doi.org/10.1136/vr.100.12.243

3. Woo, P. C., Lau, S. K., Lam, C. S., Lau, C. C., Tsang, A. K., Lau, J. H., Bai, R., Teng, J. L., Tsang, C. C., Wang, M., Zheng, B. J., Chan, K. H., \& Yuen, K. Y. (2012). Discovery of seven novel Mammalian and avian coronaviruses in the genus deltacoronavirus supports bat coronaviruses as the gene source of alphacoronavirus and betacoronavirus and avian coronaviruses as the gene source of 
gammacoronavirus and deltacoronavirus. Journal of virology, 86(7), 3995-4008. https://doi.org/10.1128/JVI.06540-11

4. Saeng-Chuto, K., Madapong, A., Kaeoket, K., Piñeyro, P. E., Tantituvanont, A., \& Nilubol, D. (2021). Coinfection of porcine deltacoronavirus and porcine epidemic diarrhea virus increases disease severity, cell trophism and earlier upregulation of IFN- $a$ and IL12. Scientific reports, 11(1), 3040. https://doi.org/10.1038/s41598-021-82738-8

5. Zhang, H., Han, F., Shu, X., Li, Q., Ding, Q., Hao, C., Yan, X., Xu, M., \& Hu, H. (2021). Co-infection of porcine epidemic diarrhoea virus and porcine deltacoronavirus enhances the disease severity in piglets. Transboundary and emerging diseases, 10.1111/tbed.14144. Advance online publication. https://doi.org/10.1111/tbed.14144

6. Dong, N., Fang, L., Yang, H., Liu, H., Du, T., Fang, P., Wang, D., Chen, H., \& Xiao, S. (2016). Isolation, genomic characterization, and pathogenicity of a Chinese porcine deltacoronavirus strain CHN-HN-2014. Veterinary microbiology, 196, 98-106. https://doi.org/10.1016/j.vetmic.2016.10.022

7. Zhang, H., Liang, Q., Li, B., Cui, X., Wei, X., Ding, Q., Wang, Y., \& Hu, H. (2019). Prevalence, phylogenetic and evolutionary analysis of porcine deltacoronavirus in Henan province, China. Preventive veterinary medicine, 166, 8-15. https://doi.org/10.1016/j.prevetmed.2019.02.017

8. Feng, Y., Xu, Z., \& Zhu, L. (2020). Prevalence and phylogenetic analysis of porcine deltacoronavirus in Sichuan province, China. Archives of virology, 165(12), 2883-2889. https://doi.org/10.1007/s00705-02004796-z

9. Jung, K., \& Saif, L. J. (2021). Replication of porcine deltacoronavirus is limited in the gastrointestinal tract of neonatal piglets co-infected simultaneously or 16 hours prior with virulent porcine epidemic diarrhea virus. Veterinary microbiology, 261, 109206. https://doi.org/10.1016/j.vetmic.2021.109206

10. Jiao, Z., Liang, J., Yang, Y., Li, Y., Yan, Z., Hu, G., Gu, C., Hu, X., Cheng, G., Peng, G., \& Zhang, W. (2021). Coinfection of porcine deltacoronavirus and porcine epidemic diarrhea virus altered viral tropism in gastrointestinal tract in a piglet model. Virology, 558, 119-125.

https://doi.org/10.1016/j.virol.2021.03.006

11. Nowarski, R., Jackson, R., \& Flavell, R. A. (2017). The Stromal Intervention: Regulation of Immunity and Inflammation at the Epithelial-Mesenchymal Barrier. Cell, 168(3), 362-375.

https://doi.org/10.1016/j.cell.2016.11.040

12. Mahlakõiv, T., Hernandez, P., Gronke, K., Diefenbach, A., \& Staeheli, P. (2015). Leukocyte-derived IFN-a/ $\beta$ and epithelial IFN- $\lambda$ constitute a compartmentalized mucosal defense system that restricts enteric virus infections. PLoS pathogens, 11(4), e1004782. https://doi.org/10.1371/journal.ppat.1004782 
13. McCauley, H. A., \& Guasch, G. (2015). Three cheers for the goblet cell: maintaining homeostasis in mucosal epithelia. Trends in molecular medicine, 21(8), 492-503.

https://doi.org/10.1016/j.molmed.2015.06.003

14. Jung, K., \& Saif, L. J. (2017). Goblet cell depletion in small intestinal villous and crypt epithelium of conventional nursing and weaned pigs infected with porcine epidemic diarrhea virus. Research in veterinary science, $110,12-15$. https://doi.org/10.1016/j.rvsc.2016.10.009

15. Li, H., Pan, P., Su, X., Liu, S., Zhang, L., Wu, D., Li, H., Dai, M., Li, Y., Hu, C., \& Chen, J. (2017). Neutrophil Extracellular Traps Are Pathogenic in Ventilator-Induced Lung Injury and Partially Dependent on TLR4. BioMed research international, 2017, 8272504. https://doi.org/10.1155/2017/8272504

16. Newton, A. H., Cardani, A., \& Braciale, T. J. (2016). The host immune response in respiratory virus infection: balancing virus clearance and immunopathology. Seminars in immunopathology, 38(4), 471482. https://doi.org/10.1007/s00281-016-0558-0

17. Ingle, H., Peterson, S. T., \& Baldridge, M. T. (2018). Distinct Effects of Type I and III Interferons on Enteric Viruses. Viruses, 10(1), 46. https://doi.org/10.3390/v10010046

18. Temeeyasen, G., Sinha, A., Gimenez-Lirola, L. G., Zhang, J. Q., \& Piñeyro, P. E. (2018). Differential gene modulation of pattern-recognition receptor TLR and RIG-I-like and downstream mediators on intestinal mucosa of pigs infected with PEDV non S-INDEL and PEDV S-INDEL strains. Virology, 517, 188-198. https://doi.org/10.1016/j.virol.2017.11.024

19. Xu, Z., Zhong, H., Huang, S., Zhou, Q., Du, Y., Chen, L., Xue, C., \& Cao, Y. (2019). Porcine deltacoronavirus induces TLR3, IL-12, IFN- $\alpha$, IFN- $\beta$ and PKR mRNA expression in infected Peyer's patches in vivo. Veterinary microbiology, 228, 226-233. https://doi.org/10.1016/j.vetmic.2018.12.012

20. Li, L., Fu, F., Xue, M., Chen, W., Liu, J., Shi, H., Chen, J., Bu, Z., Feng, L., \& Liu, P. (2017). IFN-lambda preferably inhibits PEDV infection of porcine intestinal epithelial cells compared with IFN-alpha. Antiviral research, 140, 76-82. https://doi.org/10.1016/j.antiviral.2017.01.012

21. Tang, A., Li, C., Chen, Z., \& Li, T. (2017). Anti-CD20 monoclonal antibody combined with adenovirus vector-mediated IL-10 regulates spleen CD4+/CD8+ T cells and T-bet/GATA-3 expression in NOD mice. Molecular medicine reports, 16(4), 3974-3982. https://doi.org/10.3892/mmr.2017.7111

22. Zhang, M. J., Liu, D. J., Liu, X. L., Ge, X. Y., Jongkaewwattana, A., He, Q. G., \& Luo, R. (2019). Genomic characterization and pathogenicity of porcine deltacoronavirus strain CHN-HG-2017 from China. Archives of virology, 164(2), 413-425. https://doi.org/10.1007/s00705-018-4081-6

23. Qian, S., Zhang, W., Jia, X., Sun, Z., Zhang, Y., Xiao, Y., \& Li, Z. (2019). Isolation and Identification of Porcine Epidemic Diarrhea Virus and Its Effect on Host Natural Immune Response. Frontiers in microbiology, 10, 2272. https://doi.org/10.3389/fmicb.2019.02272 
24. Xia, L., Yang, Y., Wang, J., Jing, Y., \& Yang, Q. (2018). Impact of TGEV infection on the pig small intestine. Virology journal, 15(1), 102. https://doi.org/10.1186/s12985-018-1012-9

25. Berin M. C. (2012). Mucosal antibodies in the regulation of tolerance and allergy to foods. Seminars in immunopathology, 34(5), 633-642. https://doi.org/10.1007/s00281-012-0325-9

26. Worliczek, H. L., Buggelsheim, M., Saalmüller, A., \& Joachim, A. (2007). Porcine isosporosis: infection dynamics, pathophysiology and immunology of experimental infections. Wiener klinische Wochenschrift, 119(19-20 Suppl 3), 33-39. https://doi.org/10.1007/s00508-007-0859-3

27. Li, S., Yang, J., Zhu, Z., \& Zheng, H. (2020). Porcine Epidemic Diarrhea Virus and the Host Innate Immune Response. Pathogens (Basel, Switzerland), 9(5), 367.

https://doi.org/10.3390/pathogens9050367

28. Zhang, Q., \& Yoo, D. (2016). Immune evasion of porcine enteric coronaviruses and viral modulation of antiviral innate signaling. Virus research, 226, 128-141. https://doi.org/10.1016/j.virusres.2016.05.015

\section{Tables}

\section{Table 1. IHC staining method}

\begin{tabular}{|lllc|}
\hline First antibodies & $\begin{array}{l}\text { Repair } \\
\text { methods }\end{array}$ & $\begin{array}{l}\text { Antigen repair } \\
\text { buffers }\end{array}$ & Dilutions \\
\hline $\begin{array}{l}\text { Mouse anti-human CD3 monoclonal } \\
\text { antibody }\end{array}$ & High pressure & $\mathrm{pH}=9$ & $1: 3$ \\
$\begin{array}{l}\text { (LN10, Gene Tech) } \\
\text { Rabbit anti-mouse CD20 polyclonal antibody }\end{array}$ & High pressure & $\mathrm{pH}=6$ & $1: 600$ \\
\hline (GB11540, Servicebio) & & & \\
\hline
\end{tabular}

Table 2. The table of primer information 


\begin{tabular}{|c|c|c|c|}
\hline Gene & Primer sequence $\left(5^{\prime} \rightarrow 3^{\prime}\right)$ & Product size & Accession No. \\
\hline \multirow[t]{2}{*}{$\beta$-actin } & F: TGCGGGACATCAAGGAGAAG & $207 \mathrm{bp}$ & XM_003124280.5 \\
\hline & R: TAGTTTCGTGGATGCCGCAG & & \\
\hline \multirow[t]{2}{*}{ MAVS } & F: CCCCAGGAGAGAGTTCAAAG & $142 \mathrm{bp}$ & NM_005672763.1 \\
\hline & R: TCTGTGTCCTGCTTCTGATG & & \\
\hline \multirow[t]{2}{*}{ TLR2 } & F: CGTATCCATCAATGAACACTGC & $211 \mathrm{bp}$ & NM_213761.1 \\
\hline & R: TGTCTCCACATTACCGAGGG & & \\
\hline \multirow[t]{2}{*}{ MDA5 } & F: CAGTGTGCTAGCCTGCTCTG & $113 \mathrm{bp}$ & NM_001100194.1 \\
\hline & R: GCAGTGCCTTGTTTCCTCTC & & \\
\hline \multirow[t]{2}{*}{ TLR3 } & F: TCTCCAGGGTGTTTTCATGCC & $142 \mathrm{bp}$ & NM_001097444.1 \\
\hline & R: GCTGGATGTTGCTCAGGGAT & & \\
\hline \multirow[t]{2}{*}{ NF-kb } & F: TGGGAAAGTCACAGAAACCA & $206 \mathrm{bp}$ & NM_001048232.1 \\
\hline & R: CCAGCAGCATCTTCACATCT & & \\
\hline \multirow[t]{2}{*}{ IFN- $\lambda_{1}$} & F: GGTGCTGGCGACTGTGATG & $102 \mathrm{bp}$ & NM_001142837.1 \\
\hline & R: GATTGGAACTGGCCCATGTG & & \\
\hline \multirow[t]{2}{*}{ IRF3 } & F: AAGGTTGTCCCCATGTGTCTCCG & $93 \mathrm{bp}$ & NM_213770.1 \\
\hline & R: GGAAATGTGCAGGTCCACCGTG & & \\
\hline \multirow[t]{2}{*}{ IFN-a } & F: AGAAGCATCTGCAAGGTTCCC & $160 \mathrm{bp}$ & NM_214393.1 \\
\hline & R: TCTCCTCATTTGTGCCAGGAG & & \\
\hline
\end{tabular}

\section{Figures}

\section{Figure 1}

Changes in $\mathrm{CD}^{+} \mathrm{T}$ lymphocyte numbers in the small intestine. (a to I) $\mathrm{CD}^{+} \mathrm{T}$ lymphocyte in the small intestine were detected using immunohistochemistry. ( $m$ to o) Integrated optical density of $C D 3^{+} T$ lymphocyte in the intestine ( $m$, Duodenum; $n$, Jejunum; o, lleum). Data are presented as the mean \pm SD. ${ }^{\star} P<0.05,{ }^{*} P<0.01,{ }^{\star} * \star P<0.001$. control group (a to $\mathrm{c}$ ), PEDV-infected ( $d$ to $\mathrm{f}$ ), PDCoV-infected ( $\mathrm{g}$ to $\mathrm{i}$ ) and co-infected (j to l) 


\section{Figure 2}

Changes in B lymphocyte numbers in the small intestine. (a to I) B lymphocyte in the small intestine were detected using immunohistochemistry. These B lymphocytes that were stained using anti-CD20-HRP and counterstained with hematoxylin. ( $m$ to o) Integrated optical density of $B$ lymphocyte in the intestine ( $m$, Duodenum; $n$, Jejunum; 0 , lleum). Data are presented as the mean \pm SD. ${ }^{*} P<0.05,{ }^{\star *} P<0.01,{ }^{\star \star *} P<0.001$. control group (a to c), PEDV-infected ( $d$ to f), PDCoV-infected ( $\mathrm{g}$ to i) and co-infected ( $\mathrm{j}$ to $\mathrm{l}$ )

\section{Figure 3}

Changes in goblet cell numbers in the small intestine. (a to l) Goblet cells located in the villi and crypt of the intestine. These goblet cells were stained periodic acid schiff stain. ( $m$ to $o$ ) Changes in the number of goblet cells ( $m$, Duodenum; $n$, Jejunum; o, lleum). Data are presented as the mean $\pm S D .{ }^{*}<<0.05$, $\star \star P<0.01,{ }^{* \star *} P<0.001$. control group (a to $\mathrm{c}$ ), PEDV-infected ( $d$ to $\mathrm{f}$ ), PDCoV-infected ( $\mathrm{g}$ to $\mathrm{i}$ ) and coinfected $(j$ to $I)$

\section{Figure 4}

Changes in PRRs pathway related cytokines mRNA expression levels in the intestine. To investigate PDCoV + PEDV co-infection effect on intestinal PRRs of piglets, the expression of PRRs genes and its downstream pathway genes were analyzed by RT-qPCR. Control group (Mock, black), PEDV-infected (PEDV, gules), PDCoV-infected (PDCoV, green) and Co-infected (PDCoV+PEDV, blue) 